\title{
Travelling Wave Solutions to the Benney-Luke and the Higher-Order Improved Boussinesq Equations of Sobolev Type
}

\author{
Ömer Faruk Gözükızıl and Şamil Akçağıl \\ Department of Mathematics, Sakarya University, Sakarya, Turkey \\ Correspondence should be addressed to Ömer Faruk Gözükızıl, farukg@sakarya.edu.tr \\ Received 12 September 2012; Accepted 28 November 2012 \\ Academic Editor: Dragoş-Pătru Covei
}

Copyright (C) 2012 Ö. Gözükızıl and Ş. Akçağıl. This is an open access article distributed under the Creative Commons Attribution License, which permits unrestricted use, distribution, and reproduction in any medium, provided the original work is properly cited.

By using the tanh-coth method, we obtained some travelling wave solutions of two well-known nonlinear Sobolev type partial differential equations, namely, the Benney-Luke equation and the higher-order improved Boussinesq equation. We show that the tanh-coth method is a useful, reliable, and concise method to solve these types of equations.

\section{Introduction}

The term "Sobolev equation" is used in the Russian literature to refer to any equation with spatial derivatives on the highest order time derivative [1]. In other words, they are characterized by having mixed time and space derivatives appearing in the highest-order terms of the equation and were studied by Sobolev [2]. Equations of Sobolev type describe many physical phenomena [3-7]. In recent years considerable attention has been paid to the study of equations of Sobolev type. For more details we refer the reader to [8] and references therein.

The Benney-Luke equation is as follows:

$$
u_{t t}-u_{x x}+a u_{x x x x}-b u_{x x t t}+u_{t} u_{x x}+2 u_{x} u_{x t}=0,
$$

where $a$ and $b$ are positive numbers, such that $a-b=\sigma-1 / 3$ is a Sobolev type equation and studied for a very long time. The dimensionless parameter $\sigma$ is named the Bond number, which captures the effects of surface tension and gravity force and is a formally valid 
approximation for describing two-way water wave propagation in the presence of surface tension [9]. In [10] Pego and Quintero studied the propagation of long water waves with small amplitude. They showed that in the presence of a surface tension, the propagation of such waves is governed by (1.1), originally derived by Benney and Luke [11]. There are many studies concerning with this equation. Amongst them the stability analysis [9, 12], Cauchy problem [13-15], existence and analyticity of solutions [16], and travelling wave solutions [17] can be mentioned.

In [18], Schneider and Wayne showed that in the longwave limit the water wave problem without surface tension can be described approximately by two decoupled KdV equations. They considered a class of Boussinesq equation which models the water wave problem with surface tension as follows:

$$
-u_{x x x x t t}+u_{x x t t}-u_{t t}+u_{x x}+\mu u_{x x x x}+\left(u^{2}\right)_{x x}=0
$$

where $x, t, \mu \in \mathbb{R}$ and $u(x, t) \in \mathbb{R}$. Duruk et al. investigated the well posedness of the Cauchy problem

$$
-\beta u_{x x x x t t}+u_{x x t t}-u_{t t}+u_{x x}+(g(u))_{x x}=0, \quad x \in \mathbb{R}, t>0
$$

and showed that under certain conditions the Cauchy problem is globally well posed [19]. Nevertheless, several types of the improved Boussinesq equation were investigated by many researchers and found exact solutions by using exp-function method [20], modified extended tanh-function method [21], sine-cosine method [22], improved G'/G-expansion method [22], the standard tanh and the extended tanh method [23], and so forth.

The tanh-coth is a powerful and reliable technique for finding exact travelling wave solutions for nonlinear equations. This method has been used extensively, and it was subjected by some modifications using the Riccati equation. The main features of the tanhcoth method will be outlined in the subsequent section, and this method will be applied to the the Benney-Luke and the Higher-order improved Boussinesq equations. The main purpose of this work is to obtain travelling wave solutions of the above-mentioned equations and to show that the tanh-coth method can be easily applied to Sobolev type equations. Throughout the work, Maple is used to deal with the tedious algebraic operations.

\section{Outline of the Tanh-Coth Method}

Wazwaz has summarized the tanh method in the following manner.

(i) First consider a general form of nonlinear equation

$$
P\left(u, u_{t}, u_{x}, u_{x x}, \ldots\right)=0
$$

(ii) To find the traveling wave solution of (2.1), the wave variable $\xi=x-V t$ is introduced, so that

$$
u(x, t)=U(\mu \xi)
$$


Based on this one may use the following changes:

$$
\begin{aligned}
& \frac{\partial}{\partial t}=-V \frac{d}{d \xi^{\prime}}, \\
& \frac{\partial}{\partial x}=\mu \frac{d}{d \xi^{\prime}}, \\
& \frac{\partial^{2}}{\partial x^{2}}=\mu^{2} \frac{d^{2}}{d \xi^{2}}, \\
& \frac{\partial^{3}}{\partial x^{3}}=\mu^{3} \frac{d^{3}}{d \xi^{3}},
\end{aligned}
$$

and so on for other derivatives. Using (2.3) changes the PDE (2.1) to an ODE as follows:

$$
Q\left(U, U^{\prime}, U^{\prime \prime}, \ldots\right)=0
$$

(iii) If all terms of the resulting ODE contain derivatives in $\xi$, then by integrating this equation and by considering the constant of integration to be zero, one obtains a simplified ODE.

(iv) A new independent variable

$$
Y=\tanh (\mu \xi)
$$

is introduced that leads to the change of derivatives:

$$
\begin{aligned}
\frac{d}{d \xi}= & \mu\left(1-Y^{2}\right) \frac{d}{d Y^{\prime}} \\
\frac{d^{2}}{d \xi^{2}}= & -2 \mu^{2} Y\left(1-Y^{2}\right) \frac{d}{d Y}+\mu^{2}\left(1-Y^{2}\right)^{2} \frac{d^{2}}{d Y^{2}} \\
\frac{d^{3}}{d \xi^{3}}= & 2 \mu^{3}\left(1-Y^{2}\right)\left(3 Y^{2}-1\right) \frac{d}{d Y}-6 \mu^{3} Y\left(1-Y^{2}\right)^{2} \frac{d^{2}}{d Y^{2}}+\mu^{3}\left(1-Y^{2}\right)^{3} \frac{d^{3}}{d Y^{3}} \\
\frac{d^{4}}{d \xi^{4}}= & -8 \mu^{4} Y\left(1-Y^{2}\right)\left(3 Y^{2}-2\right) \frac{d}{d Y}+4 \mu^{4}\left(1-Y^{2}\right)^{2}\left(9 Y^{2}-2\right) \frac{d^{2}}{d Y^{2}} \\
& -12 \mu^{4} Y\left(1-Y^{2}\right)^{3} \frac{d^{3}}{d Y^{3}}+\mu^{4}\left(1-Y^{2}\right)^{4} \frac{d^{4}}{d Y^{4}}
\end{aligned}
$$

where other derivatives can be derived in a similar manner. 
(v) The ansatz of the form

$$
U(\mu \xi)=S(Y)=\sum_{k=0}^{M} a_{k} Y^{k}+\sum_{k=1}^{M} b_{k} Y^{-k}
$$

is introduced where $M$ is a positive integer, in most cases, that will be determined. If $M$ is not an integer, then a transformation formula is used to overcome this difficulty. Substituting (2.6) and (2.7) into the ODE, (2.4) yields an equation in powers of $Y$.

(vi) To determine the parameter $M$, the linear terms of highest order in the resulting equation with the highest order nonlinear terms are balanced. With $M$ determined, one collects the all coefficients of powers of $Y$ in the resulting equation where these coefficients have to vanish. This will give a system of algebraic equations involving the $a_{k}$ and $b_{k},(k=0, \ldots, M), V$, and $\mu$. Having determined these parameters, knowing that $M$ is a positive integer in most cases, and using (2.7) one obtains an analytic solution in a closed form.

\section{The Benney-Luke Equation}

The Benney-Luke equation can be written as

$$
u_{t t}-u_{x x}+a u_{x x x x}-b u_{x x t t}+u_{t} u_{x x}+2 u_{x} u_{x t}=0,
$$

where $a$ and $b$ are positive numbers such that $a-b=\sigma-1 / 3$ ( $\sigma$ is named the Bond number). In order to solve (3.1) by the tanh-coth method, we use the wave transformation $u(x, t)=U(\mu \xi)$ with wave variable $\xi=x-V t$; (3.1) takes on the form of an ordinary differential equation as follows:

$$
\left(V^{2}-1\right) U^{\prime \prime}+\left(a-b V^{2}\right) U^{\prime \prime \prime \prime}-3 V U^{\prime} U^{\prime \prime}=0
$$

Balancing the order of $U^{\prime \prime \prime \prime}$ with the order of $U^{\prime} U^{\prime \prime}$ in (3.2) we find $M=1$. Using the assumptions of the tanh-coth method (2.5)-(2.7) gives the solution in the form

$$
U(\mu \xi)=S(Y)=\sum_{k=0}^{1} a_{k} Y^{k}+\sum_{k=1}^{1} b_{k} Y^{-k}
$$


Substituting (3.3) into (3.2), we obtain a system of algebraic equations for $a_{0}, a_{1}, b_{1}$, and $V$ in the following form:

$$
\begin{aligned}
& Y^{10}:-24 b V^{2} a_{1} \mu^{4}+24 V a_{1}^{2} \mu^{3}+24 a a_{1} \mu^{4}=0 \\
& Y^{9}: 18 V a_{0} a_{1} \mu^{3}=0 \\
& Y^{8}:-40 b V^{2} a_{1} \mu^{4}-2 V^{2} a_{1} \mu^{2}+36 V a_{1}^{2} \mu^{3}-12 b_{1} V a_{1} \mu^{3}+40 a a_{1} \mu^{4}+2 a_{1} \mu^{2}=0 \\
& Y^{7}: 24 V a_{0} a_{1} \mu^{3}=0 \\
& Y^{6}:-16 b V^{2} a_{1} \mu^{4}-2 V^{2} a_{1} \mu^{2}+12 V a_{1}^{2} \mu^{3}-12 b_{1} V a_{1} \mu^{3}+16 a a_{1} \mu^{4}+2 a_{1} \mu^{2}=0 \\
& Y^{5}: 6 V a_{0} a_{1} \mu^{3}+6 V a_{0} b_{1} \mu^{3}=0 \\
& Y^{4}:-16 b V^{2} b_{1} \mu^{4}-2 V^{2} b_{1} \mu^{2}+12 V b_{1}^{2} \mu^{3}-12 a_{1} V b_{1} \mu^{3}+16 a b_{1} \mu^{4}+2 b_{1} \mu^{2}=0 \\
& Y^{3}: 24 V a_{0} b_{1} \mu^{3}=0 \\
& Y^{2}:-40 b V^{2} b_{1} \mu^{4}-2 V^{2} b_{1} \mu^{2}+36 V b_{1}^{2} \mu^{3}-12 a_{1} V b_{1} \mu^{3}+40 a b_{1} \mu^{4}+2 b_{1} \mu^{2}=0 \\
& Y^{1}: 18 V a_{0} b_{1} \mu^{3}=0 \\
& Y^{0}: 24 V b_{1}^{2} \mu^{3}+24 a b_{1} \mu^{4}-24 V^{2} b b_{1} \mu^{4}=0 .
\end{aligned}
$$

From the output of the Maple packages we find three sets of solutions:

$$
\begin{aligned}
& a_{0}=b_{1}=0, \quad a_{1}=\frac{(-a+b) \mu}{V\left(2 \mu^{2} b+1\right)}, \quad V= \pm \sqrt{\frac{2 \mu^{2} a+1}{2 \mu^{2} b+1}}, \\
& a_{0}=a_{1}=0, \quad b_{1}=\frac{(-a+b) \mu}{V\left(2 \mu^{2} b+1\right)}, \quad V= \pm \sqrt{\frac{2 \mu^{2} a+1}{2 \mu^{2} b+1}}, \\
& a_{0}=0, \quad a_{1}=b_{1}=\frac{(-a+b) \mu}{V\left(8 \mu^{2} b+1\right)}, \quad V= \pm \sqrt{\frac{8 \mu^{2} a+1}{8 \mu^{2} b+1}},
\end{aligned}
$$

where $\mu$ is left as a free parameter. The travelling wave solutions are as follows:

$$
\begin{gathered}
u_{1}(x, t)=\sqrt{\frac{2 \mu^{2} b+1}{2 \mu^{2} a+1}} \frac{(-a+b) \mu}{\left(2 \mu^{2} b+1\right)} \tanh \mu\left(x \mp \sqrt{\frac{2 \mu^{2} a+1}{2 \mu^{2} b+1}} t\right), \\
u_{2}(x, t)=\sqrt{\frac{2 \mu^{2} b+1}{2 \mu^{2} a+1}} \frac{(-a+b) \mu}{\left(2 \mu^{2} b+1\right)} \operatorname{coth} \mu\left(x \mp \sqrt{\frac{2 \mu^{2} a+1}{2 \mu^{2} b+1}} t\right), \\
u_{3}(x, t)=\sqrt{\frac{8 \mu^{2} b+1}{8 \mu^{2} a+1}} \frac{(-a+b) \mu}{\left(2 \mu^{2} b+1\right)}\left(\tanh \mu\left(x \mp \sqrt{\frac{8 \mu^{2} a+1}{8 \mu^{2} b+1}} t\right)+\operatorname{coth} \mu\left(x \mp \sqrt{\frac{8 \mu^{2} a+1}{8 \mu^{2} b+1}} t\right)\right) .
\end{gathered}
$$




\section{The Higher-Order Improved Boussinesq Equation}

We consider the Higher-order improved Boussinesq equation as follows:

$$
-\alpha u_{x x x x t t}+\beta u_{x x t t}-u_{t t}+u_{x x}+\left(u^{2}\right)_{x x}=0,
$$

where $\alpha$ and $\beta$ are arbitrary non zero real constants.

Using the wave transformation $u(x, t)=U(\mu \xi)$ with wave variable $\xi=x-V t$ then by integrating this equation and considering the constant of integration to be zero, we obtain the ODE as follows:

$$
-\alpha V^{2} U^{\prime \prime \prime \prime}+\beta V^{2} U^{\prime \prime}+\left(1-V^{2}\right) U+U^{2}=0
$$

Balancing the first term with the last term in (4.2) we find $M=4$. Using the assumptions of the tanh-coth method (2.5)-(2.7) gives the solution in the form

$$
U(\mu \xi)=S(Y)=\sum_{k=0}^{4} a_{k} Y^{k}+\sum_{k=1}^{4} b_{k} Y^{-k}
$$

Substituting (4.3) into (4.2), we obtain a system of algebraic equations for $a_{0}, a_{1}, a_{2}, a_{3}, a_{4}, b_{1}$, $b_{2}, b_{3}, b_{4}, \mu$, and $V$ in the following form:

$$
\begin{aligned}
& Y^{16}: a_{4}^{2}-840 V^{2} a_{4} \alpha \mu^{4}=0 \\
& Y^{15}: 2 a_{4} a_{3}-360 V^{2} a_{3} \alpha \mu^{4}=0 \\
& Y^{14}: 2 a_{4} a_{2}+a_{3}^{2}+20 V^{2} a_{4} \beta \mu^{2}+2080 V^{2} a_{4} \alpha \mu^{4}-120 V^{2} a_{2} \alpha \mu^{4}=0 \\
& Y^{13}: 2 a_{4} a_{1}+2 a_{3} a_{2}+12 V^{2} a_{3} \beta \mu^{2}+816 V^{2} a_{3} \alpha \mu^{4}-24 V^{2} a_{1} \alpha \mu^{4}=0 \\
& Y^{12}: 240 \alpha V^{2} a_{2} \mu^{4}+6 \beta V^{2} a_{2} \mu^{2}-1696 a_{4} \alpha V^{2} \mu^{4} \\
& \quad-32 a_{4} \beta V^{2} \mu^{2}-a_{4} V^{2}+a_{2}^{2}+a_{4}+2 a_{4} a_{0}+2 a_{3} a_{1}=0 \\
& Y^{11}: a_{3}-V^{2} a_{3}+2 a_{4} b_{1}+2 a_{3} a_{0}+2 a_{1} a_{2}-18 V^{2} a_{3} \beta \mu^{2} \\
& \quad-576 V^{2} a_{3} \alpha \mu^{4}+2 V^{2} a_{1} \beta \mu^{2}+40 V^{2} a_{1} \alpha \mu^{4}=0 \\
& Y^{10}: a_{2}-V^{2} a_{2}+2 a_{4} b_{2}+2 b_{1} a_{3}+2 a_{0} a_{2}+a_{1}^{2} \\
& \quad+12 V^{2} a_{4} \beta \mu^{2}+480 V^{2} a_{4} \alpha \mu^{4}-8 V^{2} a_{2} \beta \mu^{2}-136 V^{2} a_{2} \alpha \mu^{4}=0 \\
& Y^{9}: a_{1}-V^{2} a_{1}+2 a_{4} b_{3}+2 b_{2} a_{3}+2 b_{1} a_{2}+2 a_{0} a_{1} \\
& \quad+6 V^{2} a_{3} \beta \mu^{2}+120 V^{2} a_{3} \alpha \mu^{4}-2 V^{2} a_{1} \beta \mu^{2}-16 V^{2} a_{1} \alpha \mu^{4}=0
\end{aligned}
$$




$$
\begin{aligned}
& Y^{8}: a_{0}-V^{2} a_{0}+2 a_{4} b_{4}+2 b_{3} a_{3}+2 b_{1} a_{1}+2 b_{2} a_{2} \\
&+a_{0}^{2}-24 V^{2} a_{4} \alpha \mu^{4}+2 V^{2} b_{2} \beta \mu^{2}+16 V^{2} b_{2} \alpha \mu^{4}-24 V^{2} b_{4} \alpha \mu^{4} \\
&+2 V^{2} a_{2} \beta \mu^{2}+16 V^{2} a_{2} \alpha \mu^{4}+16 V^{2} b_{2} \alpha \mu^{4}-24 V^{2} b_{4} \alpha \mu^{4}+2 V^{2} a_{2} \beta \mu^{2}+16 V^{2} a_{2} \alpha \mu^{4}=0 \\
& Y^{7}: b_{1}- V^{2} b_{1}+2 b_{4} a_{3}+2 b_{1} a_{0}+2 b_{2} a_{1}+2 b_{3} a_{2} \\
&-2 V^{2} b_{1} \beta \mu^{2}-16 V^{2} b_{1} \alpha \mu^{4}+6 V^{2} b_{3} \beta \mu^{2}+120 V^{2} b_{3} \alpha \mu^{4}=0 \\
& Y^{6}: b_{2}- V^{2} b_{2}+2 b_{2} a_{0}+2 b_{3} a_{1}+2 b_{4} a_{2}+b_{1}^{2}-8 V^{2} b_{2} \beta \mu^{2} \\
&-136 V^{2} b_{2} \alpha \mu^{4}+12 V^{2} b_{4} \beta \mu^{2}+480 V^{2} b_{4} \alpha \mu^{4}=0 \\
& Y^{5}: b_{3}- V^{2} b_{3}+2 b_{1} b_{2}+2 b_{3} a_{0}+2 b_{4} a_{1}+2 V^{2} b_{1} \beta \mu^{2} \\
&+40 V^{2} b_{1} \alpha \mu^{4}-18 V^{2} b_{3} \beta \mu^{2}-576 V^{2} b_{3} \alpha \mu^{4}=0 \\
& Y^{4}: 240 \alpha V^{2} b_{2} \mu^{4}+6 \beta V^{2} b_{2} \mu^{2}-1696 b_{4} \alpha V^{2} \mu^{4} \\
& \quad-32 b_{4} \beta V^{2} \mu^{2}-b_{4} V^{2}+b_{2}^{2}+b_{4}+2 b_{1} b_{3}+2 b_{4} a_{0}=0 \\
& Y^{3}: 2 b_{1} b_{4}+2 b_{2} b_{3}-24 V^{2} b_{1} \alpha \mu^{4}+12 V^{2} b_{3} \beta \mu^{2}+816 V^{2} b_{3} \alpha \mu^{4}=0 \\
& Y^{2}: 2 b_{2} b_{4}+b_{3}^{2}-120 V^{2} b_{2} \alpha \mu^{4}+20 V^{2} b_{4} \beta \mu^{2}+2080 V^{2} b_{4} \alpha \mu^{4}=0 \\
& Y^{1}: 2 b_{3} b_{4}-360 V^{2} b_{3} \alpha \mu^{4}=0 \\
& Y^{0}: b_{4}^{2}-840 V^{2} b_{4} \alpha \mu^{4}=0 .
\end{aligned}
$$

Using Maple gives six sets of solutions:

$$
\begin{aligned}
& a_{0}=a_{4}=\frac{105 \beta^{2}}{2\left(-36 \beta^{2}+169 \alpha\right)}, \quad a_{2}=\frac{-105 \beta^{2}}{-36 \beta^{2}+169 \alpha}, \quad a_{1}=a_{3}=b_{1}=b_{2}=b_{3}=b_{4}=0, \\
& V= \pm \frac{13}{\sqrt{-1872 \mu^{2} \beta+169}}, \quad \mu= \pm \frac{1}{26} \sqrt{\frac{13 \beta}{\alpha}}, \\
& a_{0}=\frac{33 \beta^{2}}{2\left(36 \beta^{2}+169 \alpha\right)}, \quad a_{2}=\frac{-105 \beta^{2}}{36 \beta^{2}+169 \alpha}, \quad a_{4}=\frac{105 \beta^{2}}{2\left(36 \beta^{2}+169 \alpha\right)}, \\
& a_{1}=a_{3}=b_{1}=b_{2}=b_{3}=b_{4}=0, \\
& V= \pm \frac{13}{\sqrt{1872 \mu^{2} \beta+169}}, \quad \mu= \pm \frac{1}{26} \sqrt{\frac{13 \beta}{\alpha}},
\end{aligned}
$$




$$
\begin{aligned}
& a_{0}=\frac{105 \beta^{2}}{2\left(-36 \beta^{2}+169 \alpha\right)}, \quad b_{2}=\frac{-105 \beta^{2}}{-36 \beta^{2}+169 \alpha}, \quad b_{4}=\frac{105 \beta^{2}}{2\left(-36 \beta^{2}+169 \alpha\right)}, \\
& a_{1}=a_{2}=a_{3}=a_{4}=b_{1}=b_{3}=0 \text {, } \\
& V= \pm \frac{13}{\sqrt{-1872 \mu^{2} \beta+169}}, \quad \mu= \pm \frac{1}{26} \sqrt{\frac{13 \beta}{\alpha}}, \\
& a_{0}=\frac{33 \beta^{2}}{2\left(36 \beta^{2}+169 \alpha\right)}, \quad b_{2}=\frac{-105 \beta^{2}}{36 \beta^{2}+169 \alpha}, \quad b_{4}=\frac{105 \beta^{2}}{2\left(36 \beta^{2}+169 \alpha\right)}, \\
& a_{1}=a_{2}=a_{3}=a_{4}=b_{1}=b_{3}=0, \\
& V= \pm \frac{13}{\sqrt{1872 \mu^{2} \beta+169}}, \quad \mu= \pm \frac{1}{26} \sqrt{\frac{13 \beta}{\alpha}}, \\
& a_{0}=\frac{315 \beta^{2}}{16\left(-36 \beta^{2}+169 \alpha\right)}, \quad a_{2}=b_{2}=\frac{-105 \beta^{2}}{8\left(-36 \beta^{2}+169 \alpha\right)}, \quad a_{4}=b_{4}=\frac{105 \beta^{2}}{32\left(-36 \beta^{2}+169 \alpha\right)}, \\
& a_{1}=a_{3}=b_{1}=b_{3}=0 \text {, } \\
& V= \pm \frac{13}{\sqrt{-7488 \mu^{2} \beta+169}}, \quad \mu= \pm \frac{1}{52} \sqrt{\frac{13 \beta}{\alpha}}, \\
& a_{0}=\frac{-261 \beta^{2}}{16\left(36 \beta^{2}+169 \alpha\right)}, \quad a_{2}=b_{2}=\frac{-105 \beta^{2}}{8\left(36 \beta^{2}+169 \alpha\right)}, \quad a_{4}=b_{4}=\frac{105 \beta^{2}}{32\left(36 \beta^{2}+169 \alpha\right)}, \\
& a_{1}=a_{3}=b_{1}=b_{3}=0, \\
& V= \pm \frac{13}{\sqrt{7488 \mu^{2} \beta+169}}, \quad \mu= \pm \frac{1}{52} \sqrt{\frac{13 \beta}{\alpha}},
\end{aligned}
$$

The travelling wave solutions are as follows:

$$
\begin{aligned}
u_{1}(x, t)= & \frac{105 \beta^{2}}{2\left(-36 \beta^{2}+169 \alpha\right)}-\frac{105 \beta^{2}}{-36 \beta^{2}+169 \alpha} \tanh ^{2} \mu\left(x \mp \frac{13}{\sqrt{-1872 \mu^{2} \beta+169}} t\right) \\
u_{2}(x, t)= & \frac{33 \beta^{2}}{2\left(36 \beta^{2}+169 \alpha\right)}-\frac{105 \beta^{2}}{36 \beta^{2}+169 \alpha} \tanh ^{2} \mu\left(x \mp \frac{13}{\sqrt{1872 \mu^{2} \beta+169}} t\right) \\
& +\frac{105 \beta^{2}}{2\left(36 \beta^{2}+169 \alpha\right)} \tanh ^{4} \mu\left(x \mp \frac{13}{\sqrt{1872 \mu^{2} \beta+169}} t\right)
\end{aligned}
$$




$$
\begin{aligned}
& u_{3}(x, t)=\frac{105 \beta^{2}}{2\left(-36 \beta^{2}+169 \alpha\right)}-\frac{105 \beta^{2}}{-36 \beta^{2}+169 \alpha} \operatorname{coth}^{2} \mu\left(x \mp \frac{13}{\sqrt{-1872 \mu^{2} \beta+169}} t\right) \\
& +\frac{105 \beta^{2}}{2\left(-36 \beta^{2}+169 \alpha\right)} \operatorname{coth}^{4} \mu\left(x \mp \frac{13}{\sqrt{-1872 \mu^{2} \beta+169}} t\right) \\
& u_{4}(x, t)=\frac{33 \beta^{2}}{2\left(36 \beta^{2}+169 \alpha\right)}-\frac{105 \beta^{2}}{36 \beta^{2}+169 \alpha} \operatorname{coth}^{2} \mu\left(x \mp \frac{13}{\sqrt{1872 \mu^{2} \beta+169}} t\right) \\
& +\frac{105 \beta^{2}}{2\left(36 \beta^{2}+169 \alpha\right)} \operatorname{coth}^{4} \mu\left(x \mp \frac{13}{\sqrt{1872 \mu^{2} \beta+169}} t\right) \\
& u_{5}(x, t)=\frac{315 \beta^{2}}{16\left(-36 \beta^{2}+169 \alpha\right)}-\frac{105 \beta^{2}}{8\left(-36 \beta^{2}+169 \alpha\right)} \tanh ^{2} \mu\left(x \mp \frac{13}{\sqrt{-7488 \mu^{2} \beta+169}} t\right) \\
& -\frac{105 \beta^{2}}{8\left(-36 \beta^{2}+169 \alpha\right)} \operatorname{coth}^{2} \mu\left(x \mp \frac{13}{\sqrt{-7488 \mu^{2} \beta+169}} t\right) \\
& +\frac{105 \beta^{2}}{32\left(-36 \beta^{2}+169 \alpha\right)} \tanh ^{4} \mu\left(x \mp \frac{13}{\sqrt{-7488 \mu^{2} \beta+169}} t\right) \\
& +\frac{105 \beta^{2}}{32\left(-36 \beta^{2}+169 \alpha\right)} \operatorname{coth}^{4} \mu\left(x \mp \frac{13}{\sqrt{-7488 \mu^{2} \beta+169}} t\right) \text {, } \\
& u_{6}(x, t)=-\frac{261 \beta^{2}}{16\left(36 \beta^{2}+169 \alpha\right)}-\frac{105 \beta^{2}}{8\left(36 \beta^{2}+169 \alpha\right)} \tanh ^{2} \mu\left(x \mp \frac{13}{\sqrt{7488 \mu^{2} \beta+169}} t\right) \\
& -\frac{105 \beta^{2}}{8\left(36 \beta^{2}+169 \alpha\right)} \operatorname{coth}^{2} \mu\left(x \mp \frac{13}{\sqrt{7488 \mu^{2} \beta+169}} t\right) \\
& +\frac{105 \beta^{2}}{32\left(36 \beta^{2}+169 \alpha\right)} \tanh ^{4} \mu\left(x \mp \frac{13}{\sqrt{7488 \mu^{2} \beta+169}} t\right) \\
& +\frac{105 \beta^{2}}{32\left(36 \beta^{2}+169 \alpha\right)} \operatorname{coth}^{4} \mu\left(x \mp \frac{13}{\sqrt{7488 \mu^{2} \beta+169}} t\right) \text {. }
\end{aligned}
$$




\section{References}

[1] R. W. Carroll and R. E. Showalter, Singular and Degenerate Cauchy Problems, vol. 12 of Mathematics in Science and Engineering, Academic Press, New York, NY, USA, 1976.

[2] S. L. Sobolev, "Some new problems in mathematical physics," Izvestiya Akademii Nauk SSSR. Seriya Matematicheskaya, vol. 18, pp. 3-50, 1954.

[3] S. L. Sobolev, "On a new problem of mathematical physics," Izvestiya Akademii Nauk SSSR. Seriya Matematicheskaya, vol. 18, pp. 3-50, 1954.

[4] S. A. Gabov, New Problems of the Mathematical Theory of Waves, Fizmatlit, Moscow, Russia, 1998.

[5] M. O. Korpusov, Yu. D. Pletner, and A. G. Sveshnikov, "Unsteady waves in media with anisotropic dispersion," Zhurnal Vychislitel'noi Matematiki i Matematicheskoi Fiziki, vol. 39, no. 6, pp. 1006-1022, 1999.

[6] M. O. Korpusov and A. G. Sveshnikov, "Three-dimensional nonlinear evolution equations of pseudoparabolic type in problems of mathematical physics," Zhurnal Vychislitel'noi Matematiki $i$ Matematicheskoi Fiziki, vol. 43, no. 12, pp. 1835-1869, 2003.

[7] P. I. Naumkin and I. A. Shishmarëv, Nonlinear Nonlocal Equations in the Theory of Waves, vol. 133 of Translations of Mathematical Monographs, American Mathematical Society, Providence, RI, USA, 1994.

[8] E. I. Kařkina, P. I. Naumkin, and I. A. Shishmarëv, "The Cauchy problem for a Sobolev-type equation with a power nonlinearity," Izvestiya Akademii Nauk SSSR. Seriya Matematicheskaya, vol. 69, no. 1, pp. 61-114, 2005.

[9] J. R. Quintero and J. C. Muñoz Grajales, "Instability of solitary waves for a generalized Benney-Luke equation," Nonlinear Analysis. Theory, Methods \& Applications, vol. 68, no. 10, pp. 3009-3033, 2008.

[10] R. L. Pego and J. R. Quintero, "Two-dimensional solitary waves for a Benney-Luke equation," Physica D, vol. 132, no. 4, pp. 476-496, 1999.

[11] D. J. Benney and J. C. Luke, “On the interactions of permanent waves of finite amplitude," Journal of Mathematical Physics, vol. 43, pp. 309-313, 1964.

[12] J. R. Quintero, "Nonlinear stability of solitary waves for a 2-D Benney-Luke equation," Discrete and Continuous Dynamical Systems. Series A, vol. 13, no. 1, pp. 203-218, 2005.

[13] A. González N., "The Cauchy problem for Benney-Luke and generalized Benney-Luke equations," Differential and Integral Equations, vol. 20, no. 12, pp. 1341-1362, 2007.

[14] J. R. Quintero, "A remark on the Cauchy problem for the generalized Benney-Luke equation," Differential and Integral Equations, vol. 21, no. 9-10, pp. 859-890, 2008.

[15] S. Wang, G. Xu, and G. Chen, "Cauchy problem for the generalized Benney-Luke equation," Journal of Mathematical Physics, vol. 48, no. 7, Article ID 073521, 2007.

[16] J. R. Quintero, "Existence and analyticity of lump solutions for generalized Benney-Luke equations," Revista Colombiana de Matemáticas, vol. 36, no. 2, pp. 71-95, 2002.

[17] L. Ji-bin, "Exact traveling wave solutions to 2D-generalized Benney-Luke equation," Applied Mathematics and Mechanics, vol. 29, no. 11, pp. 1391-1398, 2008.

[18] G. Schneider and C. E. Wayne, "Kawahara dynamics in dispersive media," Physica D, vol. 152-153, pp. 384-394, 2001.

[19] N. Duruk, A. Erkip, and H. A. Erbay, "A higher-order Boussinesq equation in locally non-linear theory of one-dimensional non-local elasticity," IMA Journal of Applied Mathematics, vol. 74, no. 1, pp. 97-106, 2009.

[20] M. A. Abdou, A. A. Soliman, and S. T. El-Basyony, "New application of Exp-function method for improved Boussinesq equation," Physics Letters, Section A, vol. 369, no. 5-6, pp. 469-475, 2007.

[21] S. A. Elwakil, S. K. El-Labany, M. A. Zahran, and R. Sabry, "Modified extended tanh-function method and its applications to nonlinear equations," Applied Mathematics and Computation, vol. 161, no. 2, pp. 403-412, 2005.

[22] A. M. Wazwaz, "Nonlinear variants of the improved Boussinesq equation with compact and noncompact structures," Computers \& Mathematics with Applications, vol. 49, no. 4, pp. 565-574, 2005.

[23] J. Biazar and Z. Ayati, "Improved G' /G-expansion method and comparing with tanh-coth method," Applications and Applied Mathematics, vol. 6, no. 11, pp. 1981-1991, 2011. 


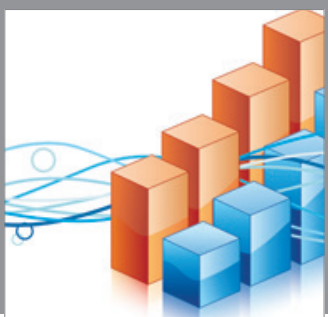

Advances in

Operations Research

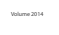

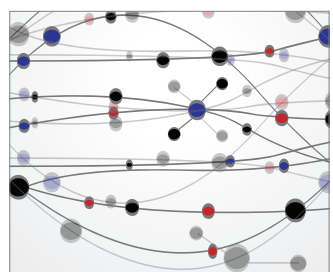

\section{The Scientific} World Journal
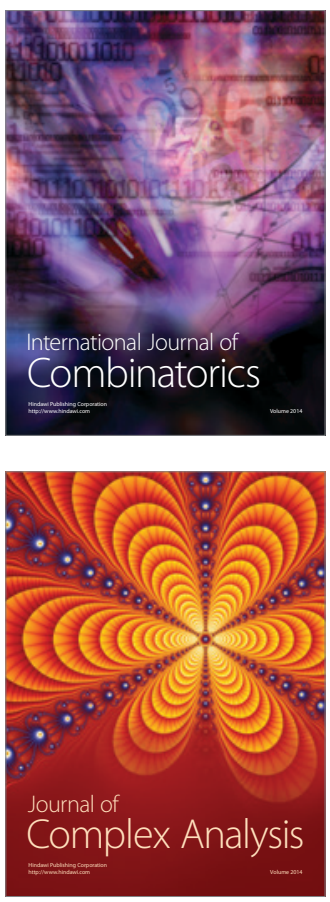

International Journal of

Mathematics and

Mathematical

Sciences
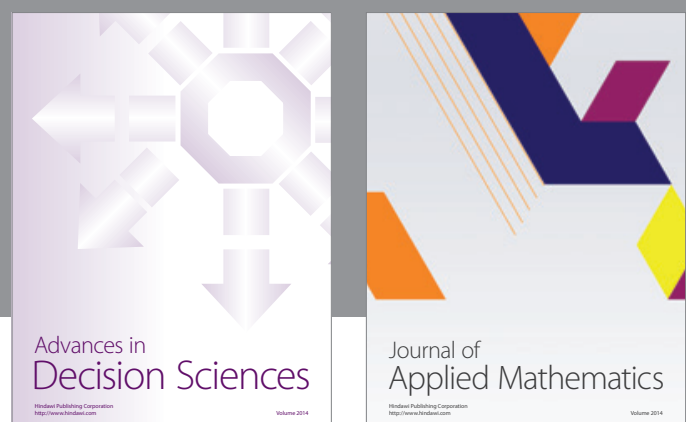

Journal of

Applied Mathematics
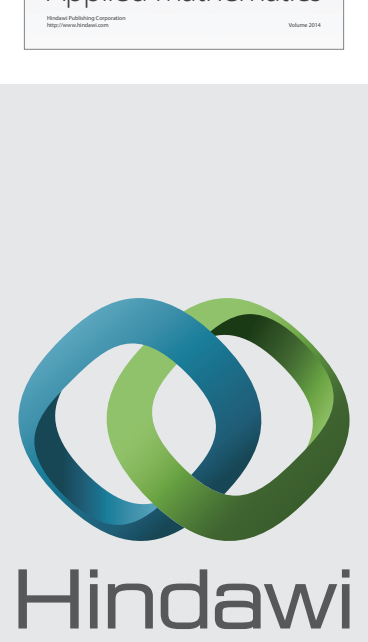

Submit your manuscripts at http://www.hindawi.com
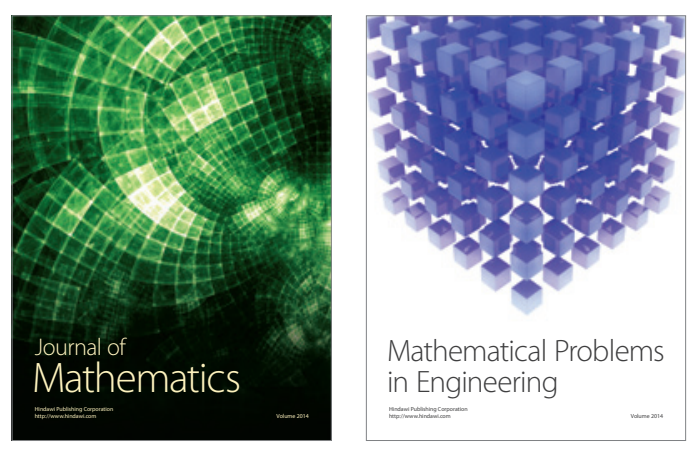

Mathematical Problems in Engineering
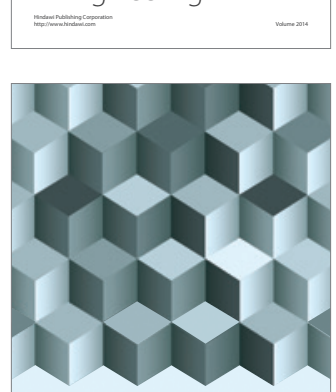

Journal of

Function Spaces
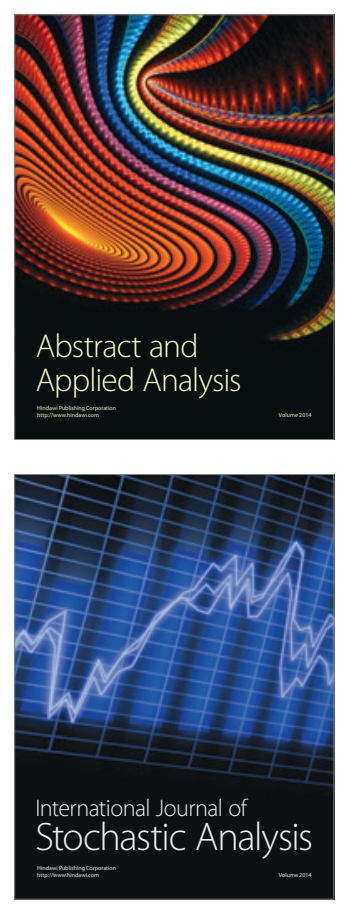

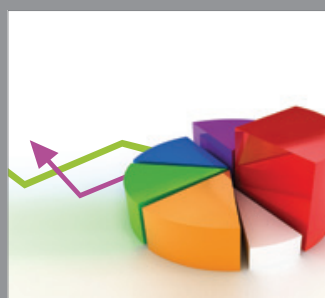

ournal of

Probability and Statistics

Promensencen
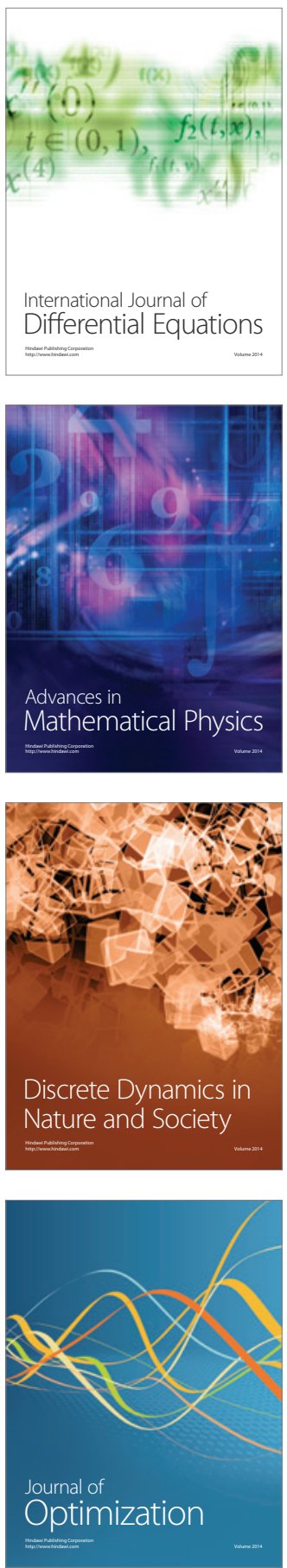